04

\title{
Импульсно-периодический диффузный разряд с автоионизацией в потоке газа
}

\author{
(C) С.Н. Буранов, В.В. Горохов, В.И. Карелин, В.Д. Селемир, А.С. Ширшин \\ Российский федеральный ядерный центр - Всероссийский научно-исследовательский институт \\ экспериментальной физики, \\ 607188 Саров, Россия \\ e-mail: shirshin@ntc.vniief.ru
}

Поступило в Редакцию 28 мая 2019 г.

В окончательной редакции 28 мая 2019 г.

Принято к публикации 12 ноября 2019 г.

\begin{abstract}
Приведены результаты исследований импульсно-периодического диффузного разряда, возбуждаемого в потоке воздуха атмосферного давления, с автоионизацией в резко неоднородном кольцевом промежутке. Разряд является источником слабоионизованной неравновесной плазмы, в которой обеспечивается эффективный синтез оксида азота. Установлено, что формирование диффузных токовых каналов происходит последовательно по длине кольцевого промежутка в направлении потока воздуха в такт с частотой высоковольтных импульсов. Анализ экспериментальных данных дает основания предполагать, что эффект автоионизации обусловлен смещением отрицательных ионов кислорода, нарабатываемых в токовых каналах, а механизмом появления затравочных электронов в новых областях пробоя является отлипание электронов от ионов кислорода.
\end{abstract}

Ключевые слова: импульсно-периодический диффузный разряд, кольцевой промежуток, автоионизация, синтез оксида азота.

DOI: 10.21883/JTF.2020.05.49175.220-19

\section{Введение}

Одним из перспективных подходов к лечению сердечно-легочных заболеваний является ингаляционная терапия оксидом азота (NO-терапия) [1,2]. В настоящее время NO синтезируют на стационарных установках, а к месту проведения лечебных процедур доставляют в баллонах. Малый срок хранения и высокая стоимость $\mathrm{NO}$, а также логистические проблемы ограничивают доступность NO-терапии, что стимулирует многочисленные исследования устройств синтеза оксида азота в электрическом разряде из воздуха непосредственно на месте проведения лечения [2]. Генераторы NO для ингаляционной терапии разрабатываются на основе дуговых и искровых разрядов [2,3]. Это объясняется тем, что в высокотемпературной плазме разрядов озон практически не нарабатывается, а концентрация двуокиси $\mathrm{NO}_{2}$ по отношению к NO низка и составляет 5-20\% [2-5]. Однако плазма таких разрядов близка к равновесной и значительная доля энергии расходуется на разогрев газа, который для фиксации NO необходимо резко охлаждать. Охлаждать газ необходимо и для целей ингаляции. Синтез NO осуществляется и в неравновесных плазмохимических системах $[6,7]$, однако техническая реализация этих систем (несамостоятельные объемные разряды, инициируемые или поддерживаемые сильноточными пучками релятивистских электронов, СВЧ-разряды в магнитном поле) достаточно сложна.

В настоящей работе приведены результаты исследований импульсно-периодического высоковольтного диф- фузного разряда атмосферного давления, обеспечивающего синтез NO в неравновесной низкотемпературной плазме. Озон в газовой смеси на выходе разрядной камеры не обнаружен, соотношение $\left[\mathrm{NO}_{2}\right] /[\mathrm{NO}]$ составляет $15 \%$ и менее, а температура выходного газа близка к комнатной. На основе такого разряда разработан аппарат для ингаляционной терапии оксидом азота $[8,9]$.

\section{Экспериментальная аппаратура}

Исследовались характеристики импульсно-периодического разряда, возбуждаемого в воздухе атмосферного давления в промежутке с резко неоднородной геометрией электрического поля. Принципиальная схема экспериментальной установки (рис. 1) включает источник питания, разрядную камеру (РК) и диагностическую аппаратуру. Источник питания 1 представляет собой инвертор с последовательным резонансным контуром и высоковольтным трансформатором [10]. По оценкам межвитковая емкость трансформатора составляет $C_{t} \approx$ $\approx 20 \mathrm{pF}$. На электродах РК источник формирует квазисинусоидальные импульсы длительностью $7 \mu$ s чередующейся полярности со скоростью нарастания напряжения $2.7 \cdot 10^{9} \mathrm{~V} / \mathrm{s}$. Частота следования импульсов от однократных до $f=10.5 \mathrm{kHz}$. Сопротивление источника $\geq 20 \mathrm{k} \Omega$.

Разрядный промежуток - кольцевой. Он образован отрезком трубы 2 внутренним диаметром $60 \mathrm{~mm}$ из дюралюминия и коаксиально расположенным диском 3 диаметром $52 \mathrm{~mm}$ из нержавеющей стали. Толщина 


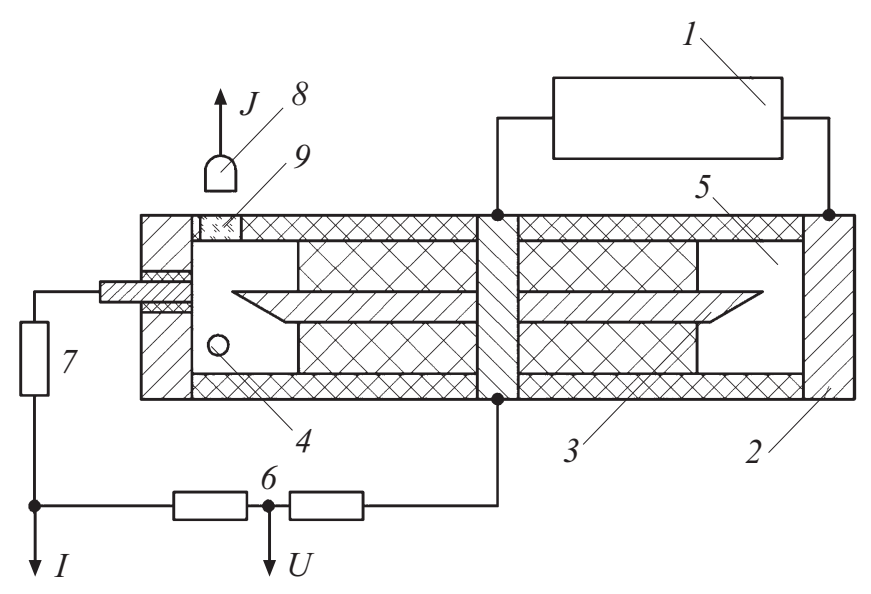

Рис. 1. Принципиальная схема экспериментального стенда (пояснение в тексте).

диска $3 \mathrm{~mm}$, угол заострения $30^{\circ}$, радиус заострения $\sim 0.1 \mathrm{~mm}$. Межэлектродное расстояние равно $4 \mathrm{~mm}$. Емкость камеры $C_{k} \approx 10 \mathrm{pF}$. Боковые фланцы камеры изготовлены из оргстекла. В экспериментах использовался осушенный воздух. Ввод воздуха в кольцевой промежуток и его вывод производился через отверстия 4,5 по касательным. Расход воздуха 1 1/min. Исследования проводились при комнатной температуре.

В экспериментах регистрировались напряжение $U$ между электродами, ток разряда $I$, интенсивность излучения $J$, спектр разряда, концентрации $\mathrm{NO}, \mathrm{NO}_{2}$ и $\mathrm{O}_{3}$. Внешний вид разряда фотографировался. Измерения временных и амплитудных характеристик сигналов производились с помощью осциллографа с полосой пропускания $500 \mathrm{MHz}$ и скоростью оцифровки $2 \mathrm{Gs} / \mathrm{s}$. Для регистрации напряжения использовался малоиндуктивный делитель 6 с полосой пропускания $75 \mathrm{MHz}$. Ток разряда измерялся шунтом 7 с измерительной площадки диаметром $2 \mathrm{~mm}$, выделенной на трубе. Чтобы обеспечить одинаковый уровень площадки относительно электрода, вся конструкция после сборки полировалась. Шунт изготовлен из высокочастотного резистора, помещенного в обратный токопровод. Временное разрешение шунта: время нарастания импульса $2.5 \mathrm{~ns}$, полуширина импульса на полувысоте 5 ns. Фотографирование осуществлялось фотоаппаратом Canon EOS 400D. Экспозиция $t_{f}$ фотоаппарата варьировалась. Интенсивность излучения $J$ регистрировалась в области $\lambda=260-900 \mathrm{~nm}$ микроканальным фотоэлектронным умножителем 8 марки С 1372. Излучение выводилось через окно 9 из кварца КУ-1 во фланце камеры. Перед фотоумножителем помещались светофильтры. Временное разрешение фотоумножителя: время нарастания импульса $3 \mathrm{~ns}$, полуширина импульса на полувысоте $6 \mathrm{~ns}$. Спектр разряда регистрировался спектрометром USB 4000 „Ocean Optics“ в диапазоне $180-850 \mathrm{~nm}$ с разрешением $1.5 \mathrm{~nm}$.

Контроль температуры воздуха осуществлялся измерителем ИВТМ-7 с абсолютной погрешностью изме- рений не более $\pm 0.2^{\circ} \mathrm{C}$. Для измерения концентраций $\mathrm{NO}$ и $\mathrm{NO}_{2}$ использовались газоанализаторы АГМ-510 двух модификаций: АГМ-510 МН - для низких концентраций и АГМ-510 МВ - для высоких. Пределы допускаемой относительной погрешности газоанализаторов $\pm 10 \%$. Измерения концентрации $\mathrm{O}_{3}$ производились анализатором озона „3.02 П-Р“ с диапазоном измеряемых концентраций до $100 \mu \mathrm{g} / \mathrm{m}^{3}$. Минимальный уровень регистрируемых концентраций озона $1 \mu \mathrm{g} / \mathrm{m}^{3}$, предел основной погрешности измерений $\pm 20 \%$.

\section{Результаты экспериментов}

На рис. 2 представлены фотографии разрядного промежутка для различных экспозиций $t_{f}$. При $t_{f}$ меньших времени между последовательными импульсами напряжения $t_{i}=1 / f$ регистрируются лишь одиночные каналы (рис. 2,a). Установлено, что число изображений каналов возрастает пропорционально $t_{f}$ (рис. 2, $b, c$ ). С учетом того что время существования тока разряда (рис. 3) в канале значительно меньше $t_{i}$, это означает, что на интегральных фотографиях отображаются новые токовые каналы. Каналы формируются последовательно в направлении прохождения газа на примерно равном расстоянии друг относительно друга, постепенно перемещаясь по всему кольцевому промежутку (рис. 2,d), т.е. первый пробой задает начальное положение, а поток газа обеспечивает последовательное перемещение пробоев по промежутку на расстояние, определяемое частотой высоковольтных импульсов и скоростью потока.

Каждый канал представляет собой столб диффузного свечения диаметром $0.3-0.4 \mathrm{~mm}$. У электродов расположены области более интенсивного свечения длиной $0.3-0.7 \mathrm{~mm}$. Расстояние между каналами зависит от частоты; при увеличении $f$ расстояние уменьшается. Установлено, что последовательное перемещение каналов наблюдается при частоте $f \geq 0.25 \mathrm{kHz}$. При меньших частотах в промежутке формируются одиночные каналы.

Типичные осциллограммы импульсов напряжения на промежутке, тока и излучения отдельного канала представлены на рис. 3. Видно, что разряд имеет две выраженные фазы: быструю и квазистационарную. Промежуток пробивается при напряжении $U_{1}=3.2-3.4 \mathrm{kV}$ на переднем фронте импульса через $\sim 1.2 \mu$ s после начала. Фронт спада импульса $\geq 50$ ns. После спада практически до нуля напряжение возрастает до $U_{2}=0.3-0.5 \mathrm{kV}$. Длительность этой фазы составляет 5.5-6 $\mu \mathrm{s}$. Импульс тока разряда представляет собой передний пик с амплитудой $I_{1}=2.7-2.8$ А длительностью 200-300 ns. За пиком следует квазистационарная фаза длительностью $4.5-5 \mu \mathrm{s}$. Ток в квазистационарной фазе $I_{2}=0.2-0.3 \mathrm{~A}$. Влияние полярности напряжения на электрические характеристики разряда не обнаружено, и до частоты $f=10.5 \mathrm{kHz}$ они лежат в обозначенных пределах. Сопротивление разряда в первой фазе $R_{1} \approx U_{1} / I_{1}=1.2 \mathrm{k} \Omega$, 

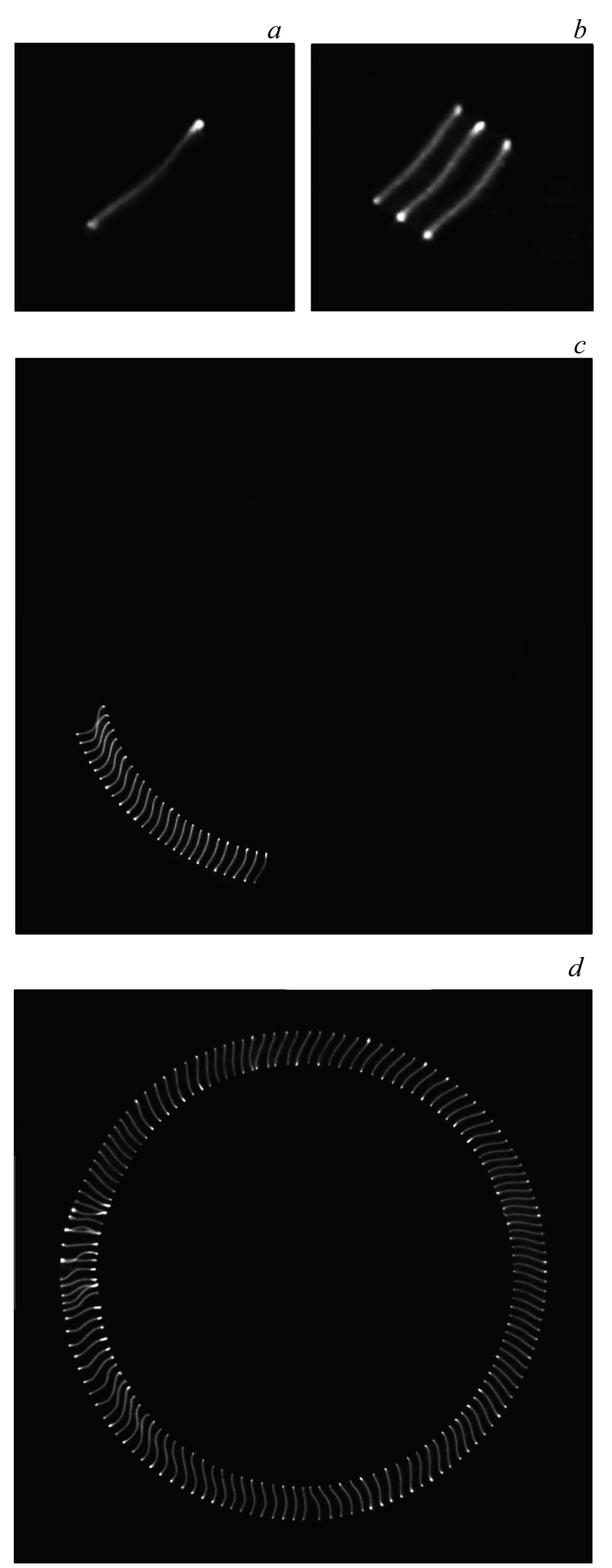

Рис. 2. Интегральные фотографии разрядного промежутка для различных экспозиций: $a-t_{f}=0.25 \mathrm{~ms}, f=2.27 \mathrm{kHz}$; $b-t_{f}=2 \mathrm{~ms}, f=1.56 \mathrm{kHz} ; c-t_{f}=16.7 \mathrm{~ms}, f=1.56 \mathrm{kHz}$; $d-t_{f}=92 \mathrm{~ms}, f=1.56 \mathrm{kHz}$. На фотографии $d$ экспозиция на $5 \mathrm{~ms}$ больше времени, за которое канал перемещается по всему кольцевому промежутку, поэтому изображения восьми каналов частично наложились друг на друга.

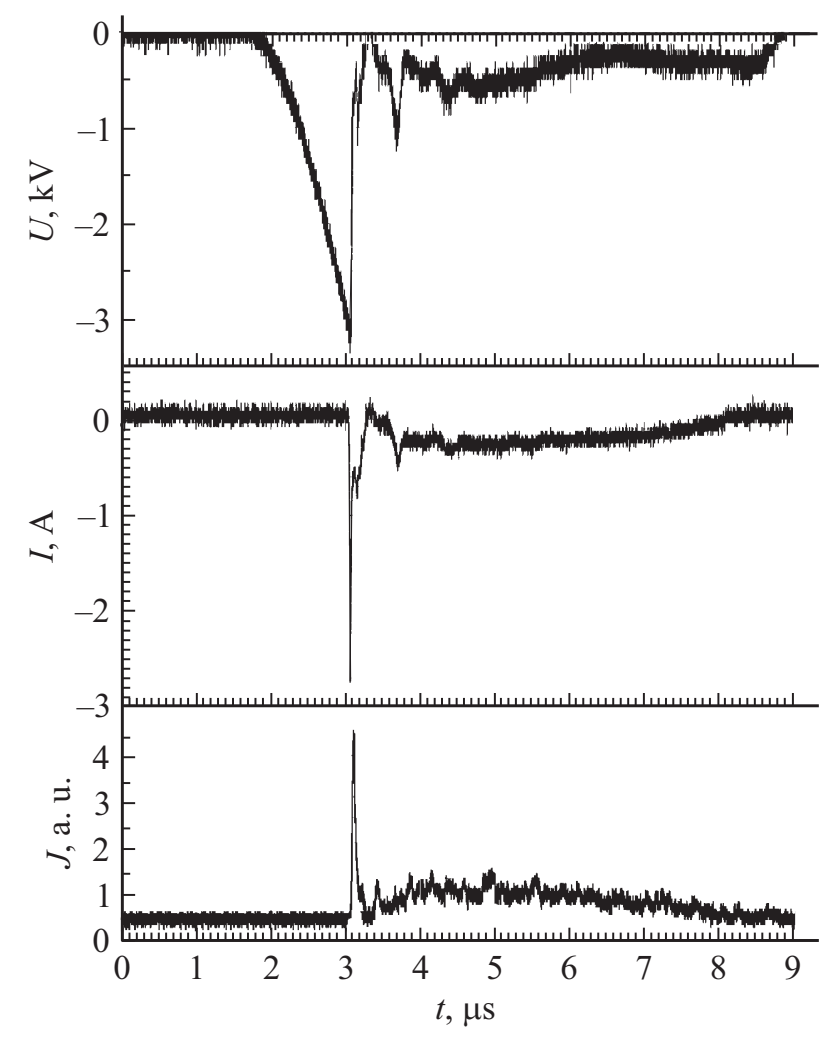

Рис. 3. Осциллограммы импульсов напряжения $U$, тока $I$ и интенсивности излучения $J$ разряда в диапазоне $\lambda=440-700 \mathrm{~nm}$ разряда.

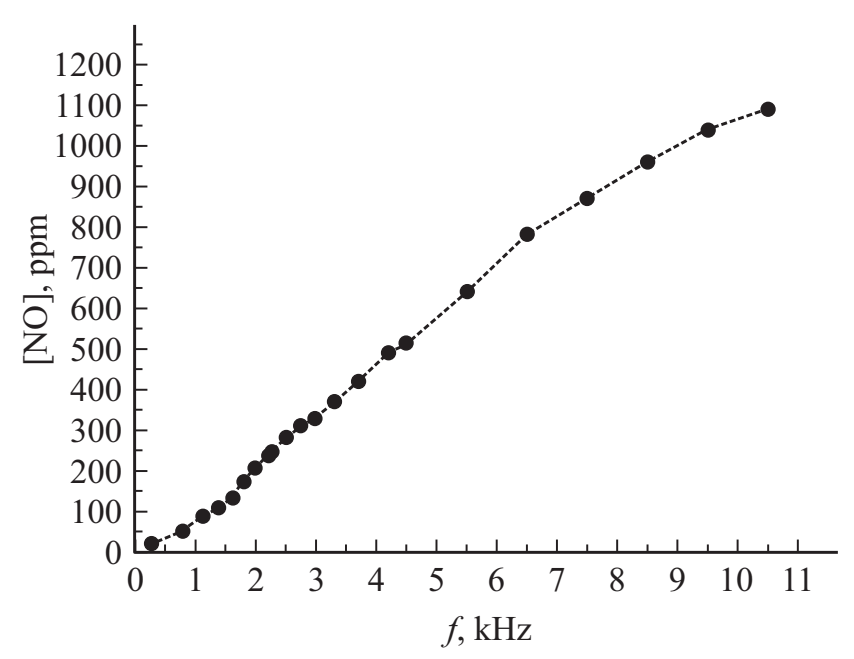

Рис. 4. Зависимость концентрации NO от частоты.

во второй $R_{2} \approx U_{2} / I_{2}=1.6 \mathrm{k} \Omega$. Форма импульса излучения практически совпадает с формой импульса тока.

В спектре разряда на фоне континуума в диапазоне 200-800 nm зарегистрированы интенсивные полосы второй положительной и первой отрицательной систем азота: $\lambda=297.7,315.9,337.1,357.7,380.5 \mathrm{~nm}$. Наиболее интенсивна полоса второй положительной системы $\lambda=337.1 \mathrm{~nm}$. 
Концентрация оксида азота зависит от частоты, причем до $f=6.5 \mathrm{kHz}$ она близка к линейной (рис. 4). Озон в выходной газовой смеси не обнаружен.

Установлено, что отношение $\left[\mathrm{NO}_{2}\right] /[\mathrm{NO}]$ от частоты зависит слабо. Так, при $f=0.25 \mathrm{kHz}$ величина $\left[\mathrm{NO}_{2}\right] /[\mathrm{NO}]=15 \%$. С повышением частоты $\left[\mathrm{NO}_{2}\right] /[\mathrm{NO}]$ уменьшается и при $f=8.5 \mathrm{kHz}$ составляет $10 \%$.

Температура выходной смеси от частоты практически не зависит. При температуре воздуха $T=24.2^{\circ} \mathrm{C}$ на вводе в разрядную камеру температура выходной смеси на конце трубки длиной $30 \mathrm{~cm}$ для $f=0.25 \mathrm{kHz}$ составляет $T=24.2^{\circ} \mathrm{C}$, а для $f=8.5 \mathrm{kHz}$ она равна $25^{\circ} \mathrm{C}$.

\section{Обсуждение}

В момент пробоя напряженность электрического поля у поверхности острой кромки $(E \geq 100 \mathrm{kV} / \mathrm{cm})$ и среднее поле по длине канала $\left(E_{1} \approx 8.3 \cdot 10 \mathrm{kV} / \mathrm{cm}\right)$ достаточны для формирования и распространения ионизационной волны, а также перекрытия промежутка при обеих полярностях [11]. Ток канала в первой фазе есть сумма токов разрядки емкостей камеры $C_{k}$, межвитковой емкости трансформатора $C_{t}$ и тока источника. Спад напряжения в этой фазе обусловлен переходным процессом разрядки емкостей $C_{k}$ и $C_{t}$.

Судя по однородности свечения столба (исключая приэлектродные области), распределение энерговыделения по длине канала примерно одинаково. Поскольку сечение канала практически постоянно, по-видимому, одинакова и напряженность поля. Оценки плотности электронов, выполненные в предположении однородности поля по длине канала, для максимума тока дают $n_{e 1}=$ $=j_{1} / e \nu_{1} \approx 3.5 \cdot 10^{15} \mathrm{~cm}^{-3}$, что соответствует степени ионизации $n_{e 1} / N \approx 1.4 \cdot 10^{-4}$. Здесь $j_{1} \approx 2.8 \cdot 10^{3} \mathrm{~A} / \mathrm{cm}^{2}-$ плотность тока, $v_{1} \approx 5 \cdot 10^{6} \mathrm{~cm} / \mathrm{s}[11]-$ дрейфовая скорость электронов, $e-$ заряд электрона, $N$ - плотность нейтралов.

В квазистационарной фазе разряда после завершения переходного процесса в контурах $C_{k}$ и $C_{t}$, ток определяется источником питания, а напряжение сопротивлением канала. Средняя напряженность поля в этой фазе разряда $E_{2} \approx 1 \mathrm{kV} / \mathrm{cm}$ недостаточна для ионизационного размножения. Разряд переходит в режим распада плазмы и сопротивление канала разряда возрастает. Удельный энерговклад в квазистационарной фазе $\sim 1.2 \mathrm{~J} / \mathrm{cm}^{3}$. По оценкам концентрация составляет $n_{e 2} \approx 2.6 \cdot 10^{15} \mathrm{~cm}^{-3}$, a $n_{e 2} / N \approx 10^{-4}$.

Обе фазы разряда характеризуются резкой неравновесностью. В соответствии с данными [11] температура электронов в первой фазе $T_{e 1} \approx 1.1-1.2 \mathrm{eV}$, а отношение $T_{e 1}$ к температуре газа $T_{e 1} / T \approx 40-45$. В квазистационарной фазе $T_{e 2} \approx 0.3-0.4 \mathrm{eV}$, а $T_{e 2} / T \approx 10-15$ и, согласно [6], параметры плазмы удовлетворяют условиям синтеза NO.

Отсутствие озона или его концентрация ниже минимального регистрируемого уровня, по-видимому, объ- ясняется, с одной стороны, существенным отличием напряженности поля и температуры электронов в исследуемом разряде от $E=20-40 \mathrm{kV} / \mathrm{cm}$ и $T_{e}=4-5 \mathrm{eV}$, при которых озон нарабатывается, с другой - его разрушением при столкновениях с нейтралами [12,13].

Вызывает интерес эффект последовательного перемещения пробоя по кольцевому промежутку. Его можно объяснить смещением продуктов синтеза разряда в направлении газового потока. Механизмом появления затравочных электронов в новой области пробоя, по-видимому, является отлипание электронов от отрицательных ионов кислорода.

Под действием потока исходного газа плазменный канал смещается, оставаясь в области кольцевого промежутка. После спада электрического поля электроны в плазме канала термализуются. Время, затрачиваемое на термализацию электронов в воздухе атмосферного давления, менее $10 \mathrm{~ns}$ [14]. После термализации электронов основными процессами, определяющими зарядовую кинетику, являются рекомбинация ионов, прилипание электронов к молекулам кислорода и отлипание электронов. Через время $t_{i}$ во время очередного высоковольтного импульса в промежутке реализуется высокое электрическое поле. К этому моменту компактное образование смещается на некоторое расстояние от места пробоя. За время $t_{i}$, соответствующее частоте $f=0.25 \mathrm{kHz}$, ниже которой в промежутке формируются одиночные каналы, концентрация положительных ионов $n_{+}$вследствие рекомбинации снижается до $n_{+}=n_{+0} /\left(1+\beta n_{+0} t_{i}\right) \approx 1.3 \cdot 10^{8} \mathrm{~cm}^{-3}$, а между прилипанием и отлипанием устанавливается приближенное динамическое равновесие [15]. Здесь $n_{+0} \approx 2.6 \cdot 10^{15} \mathrm{~cm}^{-3}-$ концентрация ионов в квазистационарной фазе, $\beta \approx 2 \cdot 10^{-6} \mathrm{~cm}^{3} / \mathrm{s}-$ коэффициент рекомбинации ионов [11]. Соответствующее равновесию отношение концентраций отрицательных ионов и электронов $n_{-} / n_{e}$ сохраняется постоянным, хотя сами концентрации уменьшаются.

Для оценки $n_{e}$ к моменту $t_{i}$ воспользуемся экспериментальными данными [15], полученными для кислорода: $n_{-} / n_{e} \approx 20$. Отметим, что для воздуха это отношение из-за наличия долгоживущих возбужденных состояний азота, по-видимому, меньше. С учетом равенства $n_{+}=n_{-}+n_{e}$ для концентрации электронов в момент $t_{i}=4 \mathrm{~ms}$ получаем $n_{e} \approx 10^{7} \mathrm{~cm}^{-3}$. При $U_{1}=3.2 \mathrm{kV}$ объем области, в которой напряженность поля обеспечивает превышение частоты ионизации над частотой потерь электронов $(\sim 30 \mathrm{kV} / \mathrm{cm})$, составляет $\sim 10^{-4} \mathrm{~cm}^{3}$ следовательно в этой области находится $\sim 10^{3}$ электронов. По-видимому, близкое к этому значению число затравочных электронов является критическим для формирования пробоя в данных условиях. При частотах больших $f=0.25 \mathrm{kHz}$ время $t_{i}$ между импульсами меньше $4 \mathrm{~ms}$ соответственно $n_{e}$ и число электронов в области размножения больше. 


\section{Заключение}

Экспериментальные данные дают основания утверждать, что диффузный импульсно-периодический разряд, возбуждаемый в потоке воздуха атмосферного давления в кольцевом промежутке с резко неоднородной геометрией электрического поля, является источником слабоионизованной неравновесной плазмы и обеспечивает эффективный синтез оксида азота. Обнаруженный эффект последовательного, в такт с частотой высоковольтных импульсов, перемещения пробоя по длине промежутка можно объяснить смещением продуктов синтеза разряда - отрицательных ионов кислорода, в направлении газового потока. Однако следует подчеркнуть, что это лишь предварительные соображения о природе импульсно-периодического пробоя в потоке газа в кольцевых резко неоднородных промежутках. Для более детальных выводов о физических процессах, обусловливающих это явление, необходимы дополнительные исследования.

\section{Конфликт интересов}

Авторы заявляют, что у них нет конфликта интересов.

\section{Список литературы}

[1] Ванин А.Ф. // Вестник РАН. 2000. № 4. С. 3-5.

[2] Malik M.A. // Plasma Chem. Plasma Process. 2016. Vol. 36. P. 737-766. DOI: $10.1007 / \mathrm{s} 11090-016-9698-1$

[3] Yu B., Muenster S., Blaesi A.H., Bloch D.B., Zapol W.M. // Sci. Transl. Med. 2015. 7(294):294ra107-294ra107. 58

[4] Namihira T., Katsuki S., Hackam R., Akiyama H., Okamoto K. // IEEE Trans Plasma Sci. 2002. Vol. 30. N 5. P. 1993-1998.

[5] Hu H., Liang H., Li J., Zhao Q., He J. // IEEE Trans Plasma Sci. 2007. Vol. 35. N 3. P. 619-625.

[6] Русанов В.Д., Фридман А.А., Шолин Г.В. // Химия плазмы. 1978. Вып. 5. С. 232-241.

[7] Русанов В.Д., Фридман А.А., Шолин Г.В. // УФН. 1981. Т. 134. Вып. 2. С. 165-235.

[8] Пат. РФ № 2593297. Способ получения газовой смеси, содержащей окись азота. Буранов С.Н., Карелин В.И., Селемир В.Д., Ширшин А.С. 2016. Бюлл. № 22.

[9] Буранов С.Н., Буянов А.Б., Воеводин С.В., Карелин В.И., Селемир В.Д., Ширшин А.С. // Биорадикалы и антиоксиданты. 2016. Т. 3. Вып. 3. С. 225-226.

[10] Буранов С.Н., Горохов В.В., Карелин В.И., Репин П.Б. // ПТЭ. 1999. Т. 42. Вып. 1. С. 134-136.

[11] Райзер Ю.П. Физика газового разряда. Долгопрудный: Издат. дом „Интеллект“, 2009. 736 с.

[12] Самойлович В.Г., Гибалов В.И., Козлов К.В. Физическая химия барьерного разряда. М.: МГУ, 1989. $176 \mathrm{c.}$

[13] Лунин В.В., Попович М.П., Ткаченко С.Н. Физическая химия озона. М.: МГУ, 1998. 480 с.

[14] Базелян Э.М., Райзер Ю.П. Искровой разряд. М.: Изд-во МФТИ, 1977. $320 \mathrm{c.}$

[15] Месси Г. Отрицательные ионы. М.: Мир, 1979. 760 с. 